\title{
Integrated population modelling reveals potential drivers of demography from partially aligned data: a case study of snowy plover declines under human stressors
}

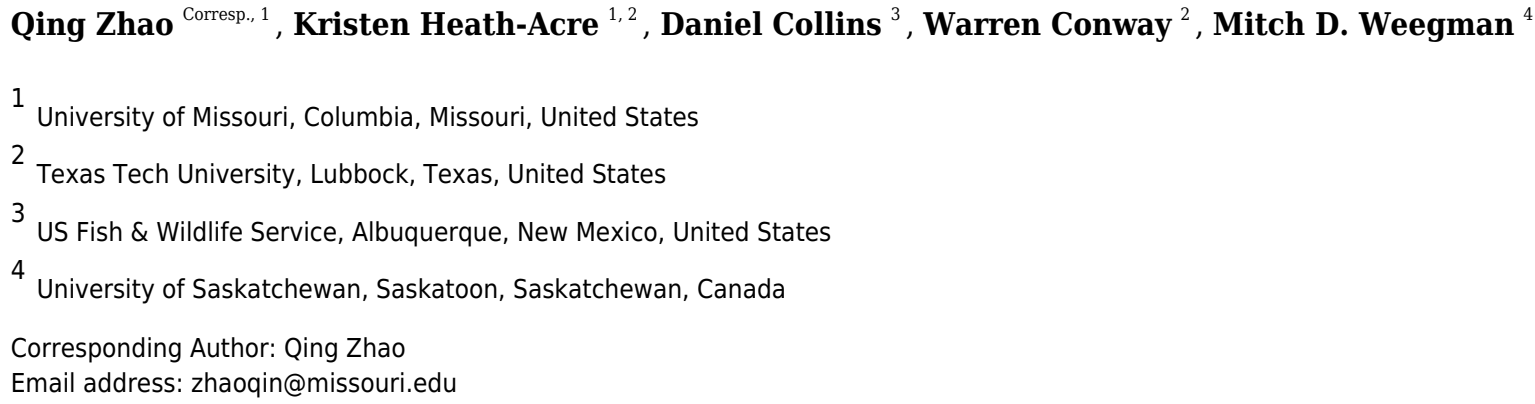

Knowledge of demography is essential for understanding wildlife population dynamics and developing appropriate conservation plans. However, population survey and demographic data (e.g., capture-recapture) are not always aligned in space and time, hindering our ability to robustly estimate population size and demographic processes. Integrated population models (IPMs) can provide inference for population dynamics with poorly aligned but jointly analysed population and demographic data. In this study, we used an IPM to analyse partially aligned population and demographic data of a migratory shorebird species, the snowy plover (Charadrius nivosus). Snowy plover populations have declined dramatically during the last two decades, yet the demographic mechanisms and environmental drivers of these declines remain poorly understood, hindering development of appropriate conservation strategies. We analysed 21 years (1998-2018) of partially aligned population survey, nest survey, and capture-recapture-resight data in three snowy plover populations (i.e., Texas, New Mexico, Oklahoma) in the Southern Great Plains of the US. By using IPMs we aimed to achieve better precision while evaluating the effects of wetland habitat and climatic factors (minimum temperature, wind speed) on snowy plover demography. Our IPM provided reasonable precision for productivity measures even with missing data, but population and survival estimates had greater uncertainty in years without corresponding data. Our model also uncovered the complex relationships between wetland habitat, climate, and demography with reasonable precision. Wetland habitat had positive effects on snowy plover productivity (i.e., clutch size and clutch fate), indicating the importance of protecting wetland habitat under climate change and other human stressors for the conservation of this species. We also found a positive effect of minimum temperature on snowy plover productivity, indicating potential benefits of warmth during 
night on their population. Based on our results, we suggest prioritizing population and capture-recapture surveys for understanding population dynamics and underlying demographic processes when data collection is limited by time and/or financial resources. Our modelling approach can be used to allocate limited conservation resources for evidence-based decision-making. 
1 Integrated population modelling reveals potential drivers of demography from partially

2 aligned data: a case study of snowy plover declines under human stressors

3

4 Qing Zhao ${ }^{1}$, Kristen Heath-Acre ${ }^{1,2}$, Dan Collins ${ }^{3}$, Warren Conway ${ }^{2}$, and Mitch D. Weegman ${ }^{4}$

$5{ }^{1}$ School of Natural Resources, University of Missouri, Columbia, Missouri, USA

$6 \quad{ }^{2}$ Department of Natural Resources Management, Texas Tech University, Lubbock, Texas, USA

7 3US Fish and Wildlife Service, Migratory Bird Program, Albuquerque, New Mexico, USA

84 Department of Biology, University of Saskatchewan, Saskatoon, Saskatchewan, Canada

10 Corresponding author: Qing Zhao

11 Address: School of Natural Resources, University of Missouri, 1111 E. Rollins, Columbia,

12 Missouri 65211

13 E-mail: zhaoqin@,missouri.edu 


\section{Abstract}

Knowledge of demography is essential for understanding wildlife population dynamics and developing appropriate conservation plans. However, population survey and demographic data (e.g., capture-recapture) are not always aligned in space and time, hindering our ability to robustly estimate population size and demographic processes. Integrated population models (IPMs) can provide inference for population dynamics with poorly aligned but jointly analysed population and demographic data. In this study, we used an IPM to analyse partially aligned population and demographic data of a migratory shorebird species, the snowy plover (Charadrius nivosus). Snowy plover populations have declined dramatically during the last two decades, yet the demographic mechanisms and environmental drivers of these declines remain poorly understood, hindering development of appropriate conservation strategies. We analysed 21 years (1998-2018) of partially aligned population survey, nest survey, and capture-recaptureresight data in three snowy plover populations (i.e., Texas, New Mexico, Oklahoma) in the Southern Great Plains of the US. By using IPMs we aimed to achieve better precision while evaluating the effects of wetland habitat and climatic factors (minimum temperature, wind speed) on snowy plover demography. Our IPM provided reasonable precision for productivity measures even with missing data, but population and survival estimates had greater uncertainty in years without corresponding data. Our model also uncovered the complex relationships between wetland habitat, climate, and demography with reasonable precision. Wetland habitat had positive effects on snowy plover productivity (i.e., clutch size and clutch fate), indicating the importance of protecting wetland habitat under climate change and other human stressors for the conservation of this species. We also found a positive effect of minimum temperature on snowy plover productivity, indicating potential benefits of warmth during night on their population. 
38 Based on our results, we suggest prioritizing population and capture-recapture surveys for

39 understanding population dynamics and underlying demographic processes when data collection

40 is limited by time and/or financial resources. Our modelling approach can be used to allocate

41 limited conservation resources for evidence-based decision-making.

42

43 Key words: climate change, conservation, data integration, demography, human stressor,

44 imbalanced sampling, population monitoring, wetland 
47 The knowledge of demographic processes is fundamental to learning about natural populations

\section{Introduction}

(Turchin 2003; Rockwood 2015). Due to continuous impacts of global change such as climate change and agricultural development on biodiversity patterns (Thomas et al. 2004; Foley et al. 2005; Parmesan 2006; Grimm et al. 2013) including population dynamics (Sæther, Sutherland \& Engen 2004; Reist et al. 2006; Simmonds \& Isaac 2007; Zhao et al. 2019), knowledge of demographic responses to environmental factors holds essential value in guiding conservation planning (Clark et al. 2001; Rushing et al. 2020).

It can be challenging to quantify population dynamics and underlying demographic processes with severely limited data, which are commonly encountered in population studies. Inferences about demographic processes (e.g., survival) often relies on data of marked animals (e.g., capture-recapture data; Pollock 1991; Williams, Nichols \& Conroy 2002), which can be difficult to collect. Furthermore, demography and population survey data are not always spatially and temporally aligned. Approaches that link population survey and demographic data are particularly useful when data are relatively sparse because they can potentially provide a comprehensive understanding of population dynamics and underlying demographic processes.

Integrated population models (IPMs) jointly analyse multiple types of data such as population survey, capture-recapture, and productivity information (Besbeas et al. 2002; Brooks, King \& Morgan 2004; Schaub \& Abadi 2011). These models can provide more accurate and precise parameter estimates than models that analyse each data type separately (Abadi et al. 2010; Schaub \& Abadi 2011). Furthermore, IPMs can provide estimates of some parameters without direct data via sharing of information among data types (Besbeas et al. 2002; Tavecchia et al. 2009; Schaub, Jakober \& Stauber 2013; Zhao, Boomer \& Royle 2019). Consequently, 
IPMs are particularly useful when data are sparse and unaligned or partially aligned in space and time (Schaub et al. 2007; Davis et al. 2014; Saunders et al. 2019). IPMs have largely improved our understanding of natural animal populations (Schaub \& Fletcher 2015; Ahrestani et al. 2017; Weegman et al. 2017; Zhao et al. 2019) and allow for the development of more effective and efficient conservation practices (Arnold et al. 2018; Zipkin \& Saunders 2018; Zhao et al. 2020).

Many shorebird populations are sensitive to climate change (Van de Pol et al. 2010;

Lehikoinen et al. 2013) because their key habitats (wetlands) are driven by weather and climatic factors (Sorenson et al. 1998; Sofaer et al. 2016; Zhao et al. 2016). Other types of human disturbance such as agricultural development may also lead to wetland habitat loss (Johnston 2013; Burgin, Franklin \& Hull 2016; Donnelly et al. 2020). Consequently, shorebirds are threatened by multiple human stressors. For example, numerous North American shorebird populations have declined during the past half-century, likely due to degradation, fragmentation, and other kinds of human disturbance of wetlands (Howe, Geissler \& Harrington 1989; Bart et al. 2007; Rosenberg et al. 2019). However, warming temperatures resulting from climate change may drive shorebird population dynamics by influencing incubation behaviour and partitioning of incubation duties, particularly during cold periods (e.g., at night), because warmer temperatures allow birds to reserve more energy for reproduction or survival (Van de Pol et al. 2010; Saalfeld et al. 2012). Additionally, greater wind speed during breeding seasons may increase physiological stress during incubation and accelerate water evaporation, and thus negatively impact shorebird demography and incubation success (Hilde et al. 2016).

The snowy plover (Charadrius nivosus) is a migratory shorebird species with breeding and wintering populations distributed along the Pacific Coast and Gulf Coast, as well as interior breeding populations in the Great Basin and Southern Great Plains (Page et al. 2009). Recent 
92 studies have estimated a severe decline of the interior breeding populations in the Southern Great

93 Plains (Andres et al. 2012; Saalfeld et al. 2013a; Heath 2019). Knowledge of the demographic

94 causes and potential drivers of such a decline is essential for conservation planning of this

95 species. However, knowledge gaps due to data limitations have hindered the development of

96 effective conservation strategies.

97 In this study we used an IPM to analyse 21 years (i.e., 1998-2018) of partially aligned data

98 for snowy plovers breeding within the Southern Great Plains. By using this modelling approach,

99 we first aimed to achieve better precision of population and demographic estimates. We then

100 evaluated the contributions of demographic processes to population growth. Lastly, we tested

101 hypotheses regarding the drivers of productivity and survival, including wetland habitat,

102 temperature, and wind speed. Based on our results, we provided recommendations for future

103 population monitoring and conservation planning of snowy plover, and suggested prioritization

104 of data collection schemes for conservation projects that often have limited resources.

\section{Methods}

\section{Study area}

108 Our study is located in the ecological region of the Southern Great Plains in Texas, New Mexico and Oklahoma (Figure 1), which encompasses semi-arid short and mixed grass prairie (Assal,

110 Melcher \& Carr 2015). More specifically, we studied 3 breeding populations in Texas, New

111 Mexico and Oklahoma, respectively. Study sites included 3 privately owned saline lakes (i.e., A,

112 B and C) and Muleshoe National Wildlife Refuge (NWR) in Texas, Bitter Lake NWR in New 
113 Mexico, and Salt Plains NWR in Oklahoma. Details about the study sites can be found in Heath 114 (2019).

\section{Data collection}

117 For the Texas population, surveys were conducted weekly May through July at lakes A, B and C 118 in 1998-2000, 2008-2010, and 2017-2018 (Figure 2), along transects that covered 3.2-3.5 km 119 sections of shoreline (Heath 2019). Multiple observers who have conducted the surveys were 120 well trained to have consistent survey abilities. Survey areas were consistent within each year 121 and among years. Surveys began at approximately 08:00 and lasted 1-2 hours in days without 122 abnormally high winds (i.e., wind speed $>50 \mathrm{mph}$ ) or rain. For the New Mexico population, 123 surveys were conducted biweekly and otherwise under the same protocol of the Texas 124 population, in each year from 1999 through 2018.

125 For the Oklahoma population, annual surveys were conducted on a single day in early May 126 from 2013 to 2017 at Salt Plains NWR. The entire salt flat area of Salt Plains NWR was divided 127 into a total of 668 grids that were $300 \mathrm{~m} \times 300 \mathrm{~m}$, among which 100 were randomly selected for 128 surveys. Biologists and volunteers were paired, and 10-12 grid cells were assigned to each pair to 129 survey. Surveys were conducted along $300 \mathrm{~m}$ transects while birds within $75 \mathrm{~m}$ distance from the 130 transects were counted. In addition to annual surveys, distance sampling was conducted at Salt 131 Plains NWR during May-July in 2017 and 2018. The region was divided into three sub-regions 132 (i.e., north, middle, south). The same grids of the annual survey were used, among which 9 (3 for 133 north, 2 for middle, 4 for south) were randomly selected. Surveys were again conducted along 
$134300 \mathrm{~m}$ transects, but in addition to counting birds with $75 \mathrm{~m}$ distance, the linear distance between

135 the observed birds and the transects was also recorded.

136 More details about the population surveys can be found in Saalfeld et al. (2013a) and Heath-

137 Acre et al. (2021).

138 Nest survey

139 We surveyed snowy plover nests at least once per week during the breeding season. Nests were

140 located by searching suitable habitat and observing adults incubating nests or flushing from or

141 returning to nests. The search effort was relatively consistent among study sites and years

142 (Conway, Smith \& Ray 2005). Once a nest was located, clutch size (i.e., the number of eggs) and

143 ultimately clutch fate (success or failure) were determined and recorded. Nest surveys were

144 conducted in 1999-2000, 2008-2009, and 2017-2018 at lakes A, B and C, and in 1999-2000 and

145 2008-2009 at Muleshoe NWR for the Texas population, in 2017-2018 at Bitter Lake NWR for

146 the New Mexico population, and in 2017-2018 at the Salt Plains NWR for the Oklahoma

147 population.

148 Capture-recapture-resight

149 Adult snowy plovers were captured at feeding locations using mist nets and on nests using nest

150 traps (Conway \& Smith 2000). Juveniles were captured within 24 hours of hatching when they

151 were still close to the nest and had not moved into foraging grounds, either by hand in nests or

152 with adult(s) after hatching. All captured individuals were banded with a uniquely numbered

153 U.S. Geological Survey aluminium band and a unique combination of colour bands. Blood

154 samples were collected during captures to identify sex (Saalfeld et al. 2013a). The identities of

155 banded birds were recorded during subsequent captures or population surveys (see above), 
156 yielding recapture and resighting information. The capture-recapture-resight surveys were

157 conducted in 1999-2000, 2008-2009, 2013-2014, and 2016-2018 at lakes A, B and C and

158 Muleshoe NWR for the Texas population, in 2013-2014 and 2017-2018 at the Bitter Lake NWR

159 for the New Mexico population, and in 2013-2018 at the Salt Plains NWR for the Oklahoma

160 population.

\section{Environmental data}

162 We considered Palmer drought severity index, minimum temperature, and wind speed as 163 potential drivers of snowy plover productivity and survival. Palmer drought severity index is a

164 measurement of the amount of surface water based on recent precipitation and temperature

165 (Palmer 1965). As Palmer drought severity index tends to be positively correlated with

166 precipitation and negatively correlated with maximum temperature (Figure S1), it can be used to

167 represent wetland habitat availability (i.e., a higher Palmer drought severity index value indicates

168 greater wetland habitat availability) that influences shorebird demography (Todhunter 1995;

169 Dinsmore 2008). High minimum temperature represented warmth during night, which may

170 influence snowy plover demography through energy reserves. We considered wind speed

171 because greater wind speed may increase physiological stress during incubation and thus

172 negatively influence snowy plover survival and productivity. We initially also considered actual

173 evapotranspiration, precipitation, and maximum temperature, but these variables were highly

174 correlated with Palmer drought severity index and/or minimum temperature (Figure S1), and

175 thus were not included in the model. We calculated the mean values of the above-mentioned

176 covariates during the breeding season (i.e., May-July) for each population and year (Figure S2)

177 and included them in our IPM.

178 Modelling approach 
179 We used an IPM to explain snowy plover population dynamics as a consequence of the 180 spatiotemporal variation in productivity and survival. Our IPM included three sub-models: a

181 population sub-model, a productivity sub-model, and a survival sub-model, which utilized

182 population survey, nest survey, and capture-recapture-resight data, respectively. We describe 183 each sub-model and then the overall model below.

184 Population sub-model

185 We assumed that population size in region $i$ in the first year, denoted $N_{i, 1}$, followed a $\log$-Normal 186 distribution such that $\log \left(N_{i, 1}\right) \sim \operatorname{Normal}\left(\mu_{i}^{[0]}, 0.1\right)$, in which $\mu_{i}^{[0]}$ was selected based on the 187 population survey data of the corresponding region, and a small standard deviation of 0.1 was 188 used. From the second year (i.e., $t \geq 2$ ), population size was assumed to follow a log-Normal 189 distribution such that $\log \left(N_{i, t}\right)=\log \left(N_{i, t-1} \times 0.5 \times \phi_{i, t-1}^{[A M]}+N_{i, t-1} \times 0.5 \times \phi_{i, t-1}^{[A F]}+N_{i, t-1} \times 0.5 \times \gamma_{i, t-1} \times \pi_{i, t-1}\right.$ $\left.\times 0.5 \times \phi_{i, t-1}^{[J M]}+N_{i, t-1} \times 0.5 \times \gamma_{i, t-1} \times \pi_{i, t-1} \times 0.5 \times \phi_{i, t-1}^{[J F]}\right)+\varepsilon_{i, t}^{[N]}$,

192 in which $\phi_{i, t-1}^{[A M]}, \phi_{i, t-1}^{[A F]}, \phi_{i, t-1}^{[J M]}$, and $\phi_{i, t-1}^{[J F]}$ were apparent survival of adult males, adult females, 193 juvenile males, and juvenile females, respectively, $\gamma_{i, t-1}$ was average clutch size, $\pi_{i, t-1}$ was 194 clutch fate, and $\varepsilon_{i, t}^{[N]}$ were process errors that followed a Normal distribution of mean 0 and 195 standard deviation $\sigma^{[N]}$. The process errors $\varepsilon_{i, t}^{[N]}$ represented the effects of demographic 196 stochasticity. Note that we assumed that all juveniles breed at one year old (Warriner et al. 197 1986). We also assumed that both adult sex ratio and clutch sex ratio were 1:1 (Saalfeld et al. 198 2013b). We did not consider partial nest mortality because it was rare in our study populations 
199 (Conway personal communication). We ignored immigration because we did not observe

200 movement among our study populations during our study.

201 We then linked the observed population survey data with the true but latent population size

202 by specifying an observation model that was specific to each data set that we fused in our IPM.

203 For the Texas and New Mexico populations, we assumed that population counts followed

204 Poisson log-Normal distributions such that $y_{i, t, l, k} \sim \operatorname{Poisson}\left(N_{i, t} \times \exp \left[\varepsilon_{i, t, l, k}^{\left[{ }^{[}\right]}\right] \times p^{[\operatorname{COUNT}]}\right)$, in

205 which $y_{i, t, l, k}$ was the population count of population $i$ in year $t$ at lake $l$ and on date $k, \varepsilon_{i, t, l, k}[y]$ were

206 lake- and date-specific variation in local abundance that followed a Normal distribution of mean

2070 and standard deviation $\sigma^{[y]}$. The detection probability $p^{[\operatorname{COUNT}]}$ for these surveys was assumed

208 to be a constant that equalled the detection probability at the median distance $(\bar{d}=30$ meters $)$ in

209 distance sampling (see below) such that $p^{[C O U N T]}=\exp (-1 \times \xi \times \bar{d})$.

210 For the Oklahoma population, we assumed that the grid-level (indexed by $j$ ) counts followed

211 a Poisson log-Normal distribution such that $y_{i, t, j} \sim \operatorname{Poisson}\left(\frac{N_{i, t}}{668} \times \exp \left[\varepsilon_{i, t, j}^{[y]}\right] \times p^{[\operatorname{COUNT}]}\right)$ for the

212 annual surveys from 2013 to 2017 , in which the total population size was divided by the total

213 number of grids (i.e., 668, see above), $\varepsilon_{i, t, j}^{[y]}$ represented the variation in local abundance among

214 grid $j$, and $p^{[C O U N T]}$ again was the constant detection probability for these annual surveys. We

215 also assumed $y_{i, t, j} \sim \operatorname{Poisson}\left(\frac{N_{i, t}}{668} \times \exp \left[\varepsilon_{i, t, j}^{[y]}\right] \times p_{i, t, j}^{[D I S T]}\right)$ for the distance sampling in 2017 and

216 2018. Here we had $p^{[D I S T]}=\exp \left(-1 \times \xi \times d_{i, t, j}\right)$, in which $\xi$ was a decay parameter

217 representing the assumption that detection probability would decrease when the linear distance

218 between the birds and the transect, denoted $d_{i, t, j}$, increased (Royle et al. 2004; Schmidt \&

219 Rattenbury 2018). 
221 We assumed that clutch size and fate were functions of Palmer drought severity index, minimum

222 temperature and wind speed. More specifically, we linked clutch size with these covariates using

223 a multinomial logistic regression. We considered the probability of a clutch size $n$ (denoted $\omega_{i, t}^{[n]}$ )

224 for $n=1,2$, and 3. Average clutch size was then calculated as $\gamma_{i, t}=1 \times \omega_{i, t}^{[1]}+2 \times \omega_{i, t}^{[2]}+3 \times$

$225 \omega_{i, t}^{[3]}$. We then had $\omega_{i, t}^{[n]}=\frac{\theta_{i, t}^{[n]}}{\sum_{n=1}^{3} \theta_{i, t}^{[n]}}$, in which $\theta_{i, t}^{[1]}=1$, and $\theta_{i, t}^{[2]}$ and $\theta_{i, t}^{[3]}$ were expressed as functions

226 of the above-mentioned covariates such that

227

$\log \left(\theta_{i, t}^{[2]}\right)=\alpha^{[2]}+\beta_{1}^{[2]} \times P D S I_{i, t}+\beta_{2}^{[2]} \times M I N T_{i, t}+\beta_{3}^{[2]} \times W I N D_{i, t}+\varepsilon_{i, t}^{[2]}$,

228 and

229

$\log \left(\theta_{i, t}^{[3]}\right)=\alpha^{[3]}+\beta_{1}^{[3]} \times P D S I_{i, t}+\beta_{2}^{[3]} \times M I N T_{i, t}+\beta_{3}^{[3]} \times W I N D_{i, t}+\varepsilon_{i, t}^{[3]}$,

in which $P D S I_{i, t}$ was the Palmer drought severity index for region $i$ and year $t, M I N T_{i, t}$ was

231 minimum temperature, $W I N D_{i, t}$ was wind speed, and $\varepsilon_{i, t}^{[2]}$ and $\varepsilon_{i, t}^{[3]}$ were process errors that

232 followed Normal distributions of mean 0 and standard deviations of $\sigma^{[2]}$ and $\sigma^{[3]}$, respectively.

233 Note that we used intercept and slope parameters that were the same across the regions, which

234 represented the assumption the demography-environment relationships were the same across

235 regions. We also considered regressions with region-specific intercept and slope parameters such

236 that $\log \left(\theta_{i, t}^{[2]}\right)=\alpha_{i}^{[2]}+\beta_{1, i}^{[2]} \times P D S I_{i, t}+\beta_{2, i}^{[2]} \times M I N T_{i, t}+\beta_{3, i}^{[2]} \times W I N D_{i, t}+\varepsilon_{i, t}^{[2]}$ and $\log \left(\theta_{i, t}^{[3]}\right)$

$237=\alpha_{i}^{[3]}+\beta_{1, i}^{[3]} \times P D S I_{i, t}+\beta_{2, i}^{[3]} \times M I N T_{i, t}+\beta_{3, i}^{[3]} \times W I N D_{i, t}+\varepsilon_{i, t}^{[3]}$ to allow for region-specific

238 demography-environment relationships.

239 We linked clutch fate with the same covariates using a logistic regression such that 
240 241

242 in which process errors $\varepsilon_{i, t}^{[f]}$ followed a Normal distribution with mean 0 and standard deviations

243 of $\sigma^{[f]}$. As in the regressions of clutch size, we also formed a regression with region-specific

244 intercept and slope parameters for clutch fate.

\section{Survival sub-model}

$\operatorname{logit}\left(\phi_{i, t-1}^{[C]}\right)=\alpha^{[C]}+\beta_{1}^{[C]} \times P D S I_{i, t}+\beta_{2}^{[C]} \times M I N T_{i, t}+\beta_{3}^{[C]} \times W I N D_{i, t}+\varepsilon_{i, t}^{[C]}$,

249 in which $\phi_{i, t-1}^{[C]}$ was the apparent survival of cohort $C$ (i.e., adult male, adult female, juvenile

250 male, juvenile female) in region $i$ and year $t$, and process errors $\varepsilon_{i, t}^{[C]}$ followed a Normal

251 distribution with mean 0 and standard deviations of $\sigma^{[C]}$. We again considered a regression with 252 region-specific intercept and slope parameters for apparent survival.

253 We also estimated the probability of recapture $\left(p^{[R E C]}\right)$ and resighting $\left(p^{[R E S]}\right)$. The

254 likelihood of the individual encounter history data was then calculated using $\phi_{i, t-1}^{[C]}, p^{[R E C]}$, and $255 p^{[R E S]}$ values.

\section{Model implementation}

257 We implemented the IPM in a hierarchical Bayesian framework with posterior distributions 258 obtained by Markov chain Monte Carlo (MCMC) computing in the software JAGS (Plummer 259 2003), which was called from R (R Development Core Team 2013) through the package 
260 “jagsUI” (Kellner 2015). We used vague priors Normal(0, 100) for any intercept and slope

261 parameters, $\operatorname{Gamma}(0.01,0.01)$ for any precision parameters and the decay parameter in distance

262 sampling, and Uniform $(0,1)$ for capture and resighting probability parameters. We used 4 chains

263 with 300,000 iterations including 50,000 burn-in and thinned by 100 , yielding 10,000 posterior

264 samples for each parameter. We checked the convergence of the MCMC computing using R-hat

265 statistics and Gelman-Rubin diagnostics (Brooks \& Gelman 1998). The R-hat statistics for each

266 parameter were $\leq 1.02$, and the chains were well mixed.

\section{Post-modelling analysis}

268 We conducted posterior predictive checks to examine model fit (Gelman 2004). We used Chi-

269 square statistics as the discrepancy measures for the population survey and distance sampling

270 data (Gelman et al. 1996). We used the Freeman-Tukey statistic as the discrepancy measures for

271 the clutch size, clutch fate, and capture-recapture-resighting data (Brooks et al. 2000). We

272 calculated these discrepancy measures for both the actual data and simulated data using the full

273 posterior samples. We then calculated posterior predictive $P$-values as the proportion of

274 discrepancy measures that were larger for the simulated data than for the actual data. A posterior

275 predictive $P$-value that is close to 0.5 indicates a good model fit.

276 We conducted a hierarchical partitioning analysis (Mac Nally 1996) to understand the

277 relative contributions of demographic parameters in describing population growth rates (Zhao,

278 Boomer \& Royle 2019). Hierarchical partitioning is based on multiple linear regressions, in

279 which population growth (i.e., $\frac{N_{i, t}}{N_{i, t-1}}$ ) was the response variable and demographic parameters were

280 the predictors. This approach considers all possible models, each of which corresponds to a given

281 combination of predictors. For each model, the joint contribution of the predictors is calculated. 
282 With such information, hierarchical partitioning allowed us to calculate the relative independent 283 contributions of each demographic parameter on population growth. We used the full posterior 284 samples to conduct these analyses to account for uncertainty in parameter estimates.

285 We calculated and reported standardized effect sizes (SES) for slope parameters representing 286 the effects of environmental covariates on demographic parameters. The standardized effect size 287 was defined as the absolute ratio of posterior mean to the width of $80 \%$ Credible Interval. We

288 289 290

291

292

293 294 295

296

297 298

299 300

301

302 considered the effect of a covariate "strong" when SES was $>0.6$, "moderate" when SES was 0.3-0.6, and "little to no effect" when SES was $<0.3$.

\section{Results}

\section{Population and demographic estimates}

Posterior predictive check indicated good model fit for all sub-models (population survey and distance sampling: posterior predictive $P$-value $=0.49$; clutch size: $P$-value $=0.42$; clutch fate: $P$-value $=0.58$; capture-recapture-resighting: $P$-value $=0.62)$. larger population size (e.g., mean 1815.0, 80\% Credible Interval [C.I.] 1590.1, 2081.2 in 2017) than the Texas (mean 83.7, 80\% C.I. 75.2, 93.4 in 2017) and New Mexico populations (mean 23.9, 80\% C.I. 20.8, 27.4 in 2017). However, the trend of the Oklahoma population was less clear due to the lack of population survey data in the early years. For the population surveys, detection probability averaged 0.999 (80\% CI $0.983,1.000)$, indicating nearly perfect detection. 
303 For the distance sampling, the decay parameter averaged 0.020 (80\% CI $0.000,0.047)$, indicating

304 weak decrease in detection probability when distance increased.

305 Average clutch size (Texas: mean 2.63, 80\% C.I. 2.03, 2.97; New Mexico: mean 2.67, 80\%

306 C.I. 2.03, 2.97; Oklahoma: mean 2.62, 80\% C.I. 2.00, 2.98) and clutch fate (Texas: mean 0.37, 307 80\% C.I. 0.09, 0.84; New Mexico: mean 0.40, 80\% C.I. 0.08, 0.83; Oklahoma: mean 0.40, 80\% 308 C.I. 0.05, 0.92) were similar among populations (Figure 4). Apparent survival of each cohort was 309 also similar among populations, but adult female (Texas: mean 0.75, 80\% C.I. 0.19, 0.99; New 310 Mexico: mean 0.78, 80\% C.I. 0.18, 0.99; Oklahoma: mean 0.75, 80\% C.I. 0.13, 0.99) and adult 311 male (Texas: mean 0.78, 80\% C.I. 0.16, 0.99; New Mexico: mean 0.79, 80\% C.I. 0.15, 0.99;

312 Oklahoma: mean $0.74,80 \%$ C.I. $0.16,0.99$ ) has on average greater survival than juvenile female 313 (Texas: mean 0.17, 80\% C.I. 0.00, 0.88; New Mexico: mean 0.14, 80\% C.I. 0.00, 0.89;

314 Oklahoma: mean 0.10, 80\% C.I. 0.00, 0.89) and juvenile male (Texas: mean 0.07, 80\% C.I. 0.00, 315 0.74; New Mexico: mean 0.09, 80\% C.I. 0.00, 0.69; Oklahoma: mean 0.06, 80\% C.I. 0.00, 0.66; 316 Figure 5) in all study populations, with high uncertainty.

Demographic contributions on population growth

318 Demographic parameters considered in our IPM had similar but uncertain contributions to 319 population growth rates (clutch size: mean $8.8 \%$, $80 \%$ C.I. $2.0 \%, 31.5 \%$, clutch fate: mean $10.5 \%, 80 \%$ C.I. $2.5 \%, 37.8 \%$, adult female survival: mean $12.2 \%, 80 \%$ C.I. $2.2 \%, 45.8 \%$, adult male survival: mean $12.1 \%, 80 \%$ C.I. $2.2 \%$, $45.6 \%$, juvenile female survival: mean 10.9\%, $80 \%$ 
324 The results from the IPM with region-specific intercept and slope parameters showed little 325 regional variation in demography-environment relationships (Figure S3 \& S4). Therefore, here 326 we report the results of the model with universal intercept and slope parameters. higher wetland habitat availability) had a moderate positive effect on the probability of a clutch size of 2 (mean $0.71,80 \%$ C.I. $-0.29,1.91, \mathrm{SES}=0.32$ ), a strong positive effect on the probability of a clutch size of 3 (mean $1.59,80 \%$ C.I. $0.56,2.91, \mathrm{SES}=0.68)$, and a strong positive effect on clutch fate (mean 1.77, 80\% C.I. 1.27, 2.33, SES = 1.67; Figure $7 \& 8$ ).

Minimum temperature had little to no effect on the probability of a clutch size of 2 (mean 0.23 , $80 \%$ C.I. $-0.26,0.78, \mathrm{SES}=0.22$ ), a strong positive effect on the probability of a clutch size of 3 (mean $0.82,80 \%$ C.I. $0.34,1.37, \mathrm{SES}=0.80$ ), and a strong positive effect on clutch fate (mean 0.99, $80 \%$ C.I. $0.70,1.28$, SES = 1.69; Figure $7 \& 8$ ). Wind speed had a moderate positive effect on clutch fate (mean $0.38,80 \%$ C.I. $0.06,0.80, \mathrm{SES}=0.52$ ), but had little to no effect on the probability of a clutch size of 2 (mean $0.09,80 \%$ C.I. $-0.56,0.80, \mathrm{SES}=0.06)$ or 3 (mean 0.12 , $80 \%$ C.I. $-0.51,0.84$, SES = 0.09; Figure 7).

Palmer drought severity index had little to no effect on adult female survival (mean 0.45 , $80 \%$ C.I. $-0.45,1.28, \mathrm{SES}=0.26)$ and adult male survival (mean $0.21,80 \%$ C.I. $-0.99,1.06$, $\mathrm{SES}=0.10$ ), but had moderate positive effects on juvenile female survival (mean $1.12,80 \%$ C.I. $0.13,3.22, \mathrm{SES}=0.36)$ and juvenile male survival (mean $0.70,80 \%$ C.I. $-0.30,1.84, \mathrm{SES}=$ 0.33). Minimum temperature (adult female: mean 0.00, $80 \%$ C.I. $-0.66,0.75$,SES $=0.00$; adult male: mean-0.35, 80\% C.I.-1.24, 0.35, SES = 0.22; juvenile female: mean 0.18, 80\% C.I. -0.67 , $1.88, \mathrm{SES}=0.07$; juvenile male: mean $-0.39,80 \%$ C.I. $-1.84,0.49, \mathrm{SES}=0.17$ ) and wind speed (adult female: mean $-0.06,80 \%$ C.I. $-0.75,0.57, \mathrm{SES}=0.05$; adult male: mean $-0.07,80 \%$ 
347 C.I. $-0.78,0.55$, SES $=0.05$; juvenile female: mean 0.04, 80\% C.I. $-0.65,0.77$, SES = 0.03;

348 juvenile male: mean-0.02, $80 \%$ C.I. $-0.71,0.66, \mathrm{SES}=0.02$ ) had little to no effect on survival 349 (Figure 9).

350

351

\section{Discussion}

352

353

354

355

356

357

358

359

360

361

362

363

364

365

366

367

368

Our IPM provided reasonable precision for productivity estimates and uncovered the complex relationships between wetland habitat conditions, climate, and demography with partially aligned data. Our results showed that wetland habitat may have positively impacted productivity of snowy plover, indicating the importance of protecting wetland habitat for the conservation of this migratory shorebird that breeds in a semi-arid environment. Our results also showed that minimum temperature may have positively influenced productivity. We recommend to prioritize data collection on population and capture-recapture surveys for understanding population dynamics and underlying demographic processes when data collection is limited by time and/or financial resource.

\section{Analysis of partially aligned data}

IPMs have been increasingly used in understanding population dynamics and underlying demographic processes due to their capability of estimating parameters with unbalanced data (Schaub et al. 2007; Davis et al. 2014; Saunders et al. 2019) or even without specific data (Besbeas et al. 2002; Zhao, Boomer \& Royle 2019), at no substantial cost to bias or precision of parameter estimates (Weegman et al. 2020). It is straightforward to analyse partially aligned data with IPMs under the hierarchical Bayesian framework as the modelling approach allows for missing values in most, if not all, parts of the model. 
Furthermore, it is feasible to incorporate information-borrowing mechanisms among regions

370

371

372

373

374

375

376

377

378

379

380

381

382

383

384

385

386

387

388

389

390

391

in IPMs that is particularly important when data are sparse and/or partially aligned. Borrowing

information can be achieved by using universal or region-specific slope parameters; while the

former provides a stronger information-borrowing mechanism, the latter is flexible to reflect

region-specific demography-environment relationships. Previous work revealed that the

responses of populations to environmental conditions may be region-specific (Forchhammer et

al. 1998; Williams, Ives \& Applegate 2003; Grøtan et al. 2009). While these studies often focus

on large spatial ranges that cover multiple ecological regions, Zhao, Boomer and Royle (2019)

found that demography-environment relationships tend to be similar within, but not among,

ecological regions. Because our study area lies in one ecological region (i.e., the Southern Great

Plains), it is not surprising to find that our three populations respond similarly to the

environment. Therefore, we used a model with universal demography-environment relationships,

which allows us to achieve relatively reasonable precision, particularly for productivity

estimates, for all three populations. We encourage practitioners to consider information-

borrowing approaches among populations when data are only partly aligned, such as in our

study.

For our case study, uncertainty of population and survival estimates was still high in some years due to the lack of corresponding data, even with an advanced IPM and informationborrowing. For example, Oklahoma population estimates from 1998 to 2012 had relatively high uncertainty due to lack of population survey data during this period. These results remind us that monitoring programs are still extremely important for gaining knowledge about wildlife populations, even with advantages from recent modelling techniques. 
392 Our study revealed potential environmental drivers of demography in three declining snowy

393 plover populations in the Southern Great Plains (Andres et al. 2012; Saalfeld et al. 2013a; Heath

394 2019). Our study showed that wetland habitat had a strong positive effect on snowy plover

395 productivity measures (i.e., clutch size and clutch fate) and a moderate positive effect on juvenile

396 survival. Thus, the declines in snowy plover productivity and population size can be attributed,

397 in part, to insufficient wetland habitat. Like other shorebirds, snowy plover populations rely on

398 wetland habitat (Conway, Smith \& Ray 2005), where degradation or loss in wetland habitat may

399 decrease their productivity or even survival (Saalfeld et al. 2011; Saalfeld et al. 2013a). Wetland

400 habitat loss could be driven by climate change (e.g. Sorenson et al. 1998; Sofaer et al. 2016) as

401 well as other human stressors (Johnston 2013; Burgin, Franklin \& Hull 2016; Donnelly et al.

402 2019; Donnelly et al. 2020). For example, the decline of the snowy plover population at Bitter

403 Lake NWR may be driven by the degradation of ground water sources in the Pecos River

404 ecosystem related to agricultural development (Heath 2019).

Our study also reveals a positive effect of minimum temperature on snowy plover clutch size 406 and fate. Increases in temperature are normally considered to lead to drier habitats and thus negatively impact shorebird populations. In our study area, maximum temperature is negatively correlated with wetland habitat, represented by Palmer drought severity index (Figure S1). Even though we could not evaluate the effect of maximum temperature on snowy plover demography due to this correlation, the positive effect of wetland habitat may indicate a negative impact of

411 high temperature during day time on snowy plover demography. High temperatures not only could lead to increased evapotranspiration and drought but also may create a thermally stressful

413 environment for nesting snowy plovers that necessitates incubating parents cooling eggs during 414 daylight hours (Saalfeld et al. 2012). High minimum temperature, on the other hand, represents a 
415 relatively warm condition during night, which may benefit these birds (Van de Pol et al. 2010;

416 Saalfeld et al. 2012). Further studies that are able to disentangle the multifaceted effects of

417 climatic conditions on shorebird demography and behaviours are essential for a comprehensive

418 understanding of the impacts of anticipated change on their populations, associated with climate

419 change. Despite that we predicted a negative effect of wind speed on snowy plover demography

420 (Hilde et al. 2016), we found that wind speed had a moderate positive effect on clutch fate but

421 had no effect on other demographic parameters. However, the relatively high uncertainty of the

422 effect of wind speed indicated that further investigation is needed.

423 Interestingly, our results showed that productivity and survival of all cohorts had similar

424 contributions on snowy plover population growth. Several studies showed that productivity tends

425 to vary more and also contribute more to population growth (Alisauskas et al. 2004; Cooch et al.

426 2001; Taylor et al. 2012), although these studies focused on larger birds. Survival might play a

427 more important role than productivity in smaller birds such as snowy plovers (current study) and

428 insectivores (Schaub et al. 2015). The relatively high uncertainty of population and survival

429 estimates in some years, however, may have masked the differential contributions of

430 demography on population growth, warranting further investigation.

\section{Conservation implications}

432 Conservation programs often have limited financial resources and practitioners are challenged to 433 balance monitoring and conservation priorities. Demographic data (e.g., capture-recapture) are

434 more expensive to collect than count data of unmarked populations yet are crucial for

435 understanding demographic foundations of population dynamics. Our study showed that

436 reasonable precision of productivity estimates could be achieved even though nest survey data

437 were available for only short periods. Previous researchers showed that productivity/recruitment 
438 could be estimated without direct data using IPMs (e.g., Besbeas et al. 2002; Zhao, Boomer \&

439 Royle 2019; Weegman et al. 2020). Survival estimates in Texas also had an overall reasonable

440 precision despite the gaps in capture-recapture-resight data. Survival estimates in Oklahoma,

441 however, had relatively high uncertainty during the first 15 years of our study period due to the

442 lack of capture-recapture-resight data. Population estimates had relatively reasonable precision

443 only for years with population survey data. In particular, the Oklahoma population was much

444 larger than the other two populations and thus seems particularly important for the conservation

445 of this species, which corroborates previous work (Heath 2019). Yet the estimates of the

446 Oklahoma population had high uncertainty during the early years due to the lack of data, which

447 largely hindered our ability to identify a long-term trend for this population. Overall, it seems to

448 be reasonable to prioritize limited time and financial resource on population and capture-

449 recapture surveys for monitoring population status and developing appropriate conservation

450 strategies for this species. On the other hand, it seems to be acceptable, although not preferable,

451 to only collect nest survey data in some years.

452 Despite the imbalanced data availability, our IPM provided an understanding about the 453 environmental drivers of snowy plover in the Southern Great Plains. Our study revealed that

454 snowy plover demography and thus population dynamics are driven by wetland habitat

455 conditions, indicating the importance of wetland habitat conservation under climate change and

456 other human stressors such as groundwater mining and agricultural development (Conway,

457 Smith \& Ray 2005; Heath 2019). Our study also showed that future warming may potentially

458 benefit snowy plover populations, at least in the short term (i.e., acknowledging that beyond a

459 certain point, increased temperatures will negatively influence snowy plover productivity;

460 Saalfeld et al. 2013a). Understanding the multifaceted effects of climate on animal demography 
461 is key for accurate forecasts of population responses, and thus appropriate conservation planning

462 under climate change (Clark et al. 2001; Petchey et al. 2015).

463 Taken together, IPMs lay a foundation of allocating limited conservation resources for

464 evidence-based decision-making. The benefits of IPMs are not limited to our study species, as

465 more studies should consider balancing allocation of conservation resources among different

466 types of data.

467 


\section{Acknowledgements}

469 We thank Sarah Saalfeld, Hannah Ashbaugh, Laura Duffie and all field assistants who have

470 contributed in data collection. M. Frederiksen, M. Paquet and M. Kery provided valuable

471 comments on the manuscript. The research was funded by U.S. Fish \& Wildlife Service.

472 


\section{$473 \quad$ Literature cited}

474 Abadi, F., Gimenez, O., Arlettaz, R. \& Schaub, M. (2010). An assessment of integrated population models: bias, accuracy, and violation of the assumption of independence. Ecology, 91, 7-14.

Ahrestani, F. S., Saracco, J. F., Sauer, J. R., Pardieck, K. L. \& Royle, J. A. (2017). An integrated population model for bird monitoring in North America. Ecological Applications, 27, 916-924.

Alisauskas, R. T., Traylor, J. J., Swoboda, C. J. \& Kehoe, F. P. (2004). Components of population growth rate for White-winged Scoters in Saskatchewan, Canada. Animal Biodiversity and Conservation, 27, 451-460.

Andres, B. A., Smith, P. A., Morrison, R. G., Gratto-Trevor, C. L., Brown, S. C. \& Friis, C. A. (2012). Population estimates of North American shorebirds, 2012. Wader Study Group Bulletin, 119, 178-194.

Arnold, T. W., Clark, R. G., Koons, D. N. \& Schaub, M. (2018). Integrated population models facilitate ecological understanding and improved management decisions. The Journal of Wildlife Management, 82, 266-274.

Assal, T. J., Melcher, C. P. \& Carr, N. B. (2015). Southern Great Plains Rapid Ecoregional Assessment: pre-assessment report. US Geological Survey.

Bart, J., Brown, S., Harrington, B. \& I. Guy Morrison, R. (2007). Survey trends of North American shorebirds: Population declines or shifting distributions? Journal of Avian Biology, 38, 73-82. 
494 Besbeas, P., Freeman, S. N., Morgan, B. J. \& Catchpole, E. A. (2002). Integrating mark-

495

496

497

498

499

500

501

502

503

504

505

506

507

508

509

510

511

512

513

514 recapture-recovery and census data to estimate animal abundance and demographic parameters. Biometrics, 58, 540-547.

Brooks, S.P., Catchpole, E. A., Morgan, B. J. \& Barry, S. C. (2000). On the Bayesian analysis of ring-recovery data. Biometrics, 56, 951-956.

Brooks, S., King, R. \& Morgan, B. (2004). A Bayesian approach to combining animal abundance and demographic data. Animal Biodiversity and Conservation, 27, 515-529.

Brooks, S. P. \& Gelman, A. (1998). General methods for monitoring convergence of iterative simulations. Journal of Computational and Graphical Statistics, 7, 434-455.

Burgin, S., Franklin, M. J. \& Hull, L. (2016). Wetland loss in the transition to urbanisation: a case study from Western Sydney, Australia. Wetlands, 36, 985-994.

Clark, J. S., Carpenter, S. R., Barber, M., Collins, S., Dobson, A., Foley, J. A., Lodge, D. M., Pascual, M., Pielke Jr, R. \& Pizer, W. (2001). Ecological forecasts: an emerging imperative. Science, 293, 657-660.

Conway, W. C. \& Smith, L. M. (2000). A nest trap for Snowy Plovers. North American Bird Bander, 25, 46-48.

Conway, W. C., Smith, L. M. \& Ray, J. D. (2005). Shorebird breeding biology in wetlands of the playa lakes, Texas, USA. Waterbirds, 28, 129-138.

Cooch, E., Rockwell, R.F. \& Brault, S., 2001. Retrospective analysis of demographic responses to environmental change: a lesser snow goose example. Ecological Monographs, 71, 377400. 
515 Davis, A.J., Hooten, M.B., Phillips, M.L. and Doherty Jr, P.F., 2014. An integrated modeling

516 approach to estimating Gunnison sage-grouse population dynamics: combining index and 517 demographic data. Ecology and Evolution, 4, 4247-4257.

518 Dinsmore, S. J. (2008). Influence of drought on annual survival of the Mountain Plover in 519 Montana. The Condor, 110, 45-54.

520 Donnelly, J.P., King, S.L., Silverman, N.L., Collins, D.P., Carrera-Gonzalez, E.M., Lafón-Terrazas, A. \& Moore, J.N. (2020). Climate and human water use diminish

Donnelly, J.P., Naugle, D.E., Collins, D.P., Dugger, B.D., Allred, B.W., Tack, J.D. \& Dreitz, V.J. (2019). Synchronizing conservation to seasonal wetland hydrology and waterbird migration in semi-arid landscapes. Ecosphere, 10, e02758.

527

Foley, J. A., DeFries, R., Asner, G. P., Barford, C., Bonan, G., Carpenter, S. R., Chapin, F. S., Coe, M. T., Daily, G. C. \& Gibbs, H. K. (2005). Global consequences of land use. Science, 309, 570-574.

Forchhammer, M. C., Stenseth, N. C., Post, E. \& Landvatn, R. (1998). Population dynamics of Norwegian red deer: density-dependence and climatic variation. Proceedings of the Royal Society of London. Series B: Biological Sciences, 265, 341-350.

Gelman, A., Carlin, J. P., Stern, H. S. \& Rubin, D. B. (2004) Bayesian data analysis. CRC/Chapman \& Hall, Boca Raton, USA. via realized discrepancies. Statistica Sinica, 733-760. 
537 Grimm, N. B., Chapin, F. S., Bierwagen, B., Gonzalez, P., Groffman, P. M., Luo, Y., Melton, F., 538 Nadelhoffer, K., Pairis, A. \& Raymond, P. A. (2013). The impacts of climate change on ecosystem structure and function. Frontiers in Ecology and the Environment, 11, 474-

540 482.

541 Grøtan, V., Sæther, B. E., Engen, S., Van Balen, J. H., Perdeck, A. C. \& Visser, M. E. (2009).

542

543

544

545

546

547

548

549

550

551

552

553

554

555

556

557

558

559

Spatial and temporal variation in the relative contribution of density dependence, climate variation and migration to fluctuations in the size of great tit populations. Journal of Animal Ecology, 78, 447-459.

Heath, K.M. (2019). Population trends and connectivity of snowy plovers on the Southern Great Plains of Texas, New Mexico and Oklahoma (Master's thesis). Texas Tech University Press.

Heath-Acre, K.M., Conway, W.C., Boal, C.W., Collins, D.P., Hensley, G., Johnson, W.P. and Schmidt, P.M., 2021. Detectability and Abundance of Snowy Plovers at Salt Plains National Wildlife Refuge, Oklahoma. Journal of Fish and Wildlife Management, 12, 5060. https://doi.org/10.3996/JFWM-20-041.

Høyvik Hilde, C., Pélabon, C., Guéry, L., Gabrielsen, G.W. and Descamps, S. (2016). Mind the wind: microclimate effects on incubation effort of an arctic seabird. Ecology and Evolution, 6, 1914-1921.

Howe, M. A., Geissler, P. H. \& Harrington, B. A. (1989). Population trends of North American shorebirds based on the International Shorebird Survey. Biological Conservation, 49, 185-199.

Johnston, C. A. (2013). Wetland losses due to row crop expansion in the Dakota Prairie Pothole Region. Wetlands, 33, 175-182. 
560 Kellner, K. (2015). jagsUI: a wrapper around rjags to streamline JAGS analyses. R package $561 \quad$ version, 1.

562 Lehikoinen, A., Jaatinen, K., Vähätalo, A. V., Clausen, P., Crowe, O., Deceuninck, B., Hearn,

563 R., Holt, C. A., Hornman, M. \& Keller, V. (2013). Rapid climate driven shifts in

564 wintering distributions of three common waterbird species. Global Change Biology, 19,

565 2071-2081.

566

567

568

569

570

571

572

573

574

575

576

577

578

579

580

581

582

Mac Nally, R. (1996). Hierarchical partitioning as an interpretative tool in multivariate inference. Austral Ecology, 21, 224-228.

Page, G., Warriner, J., Warriner, J. \& Paton, P. (2009). Snowy Plover. The Birds of North America Online (A. Poole, Ed.). Ithaca: Cornell Lab of Ornithology.

Palmer, W. C. (1965). Meteorological drought. US Department of Commerce, Weather Bureau.

Parmesan, C. (2006). Ecological and evolutionary responses to recent climate change. Annual Review of Ecology, Evolution, and Systematics, 37, 637-669.

Petchey, O. L., Pontarp, M., Massie, T. M., Kéfi, S., Ozgul, A., Weilenmann, M., Palamara, G. M., Altermatt, F., Matthews, B. \& Levine, J. M. (2015). The ecological forecast horizon, and examples of its uses and determinants. Ecology Letters, 18, 597-611.

Plummer, M. (2003). JAGS: A program for analysis of Bayesian graphical models using Gibbs sampling. Proceedings of the 3rd international workshop on distributed statistical computing, pp. 1-10. Vienna, Austria.

Pollock, K. H. (1991). Review papers: modeling capture, recapture, and removal statistics for estimation of demographic parameters for fish and wildlife populations: past, present, and future. Journal of the American Statistical Association, 86, 225-238.

R Development Core Team. (2013). R: A language and environment for statistical computing. 
583 Reist, J. D., Wrona, F. J., Prowse, T. D., Power, M., Dempson, J. B., Beamish, R. J., King, J. R., 584 Carmichael, T. J. \& Sawatzky, C. D. (2006). General effects of climate change on Arctic 585 fishes and fish populations. AMBIO: A Journal of the Human Environment, 35, 370-380.

586 587 588 589

Rockwood, L. L. (2015). Introduction to population ecology. John Wiley \& Sons.

Rosenberg, K. V., Dokter, A. M., Blancher, P. J., Sauer, J. R., Smith, A. C., Smith, P. A., Stanton, J. C., Panjabi, A., Helft, L. \& Parr, M. (2019). Decline of the North American avifauna. Science, 366, 120-124.

Royle, J.A., Dawson, D.K. \& Bates, S. (2004). Modeling abundance effects in distance sampling. Ecology, 85, 1591-1597.

Rushing, C. S., Rubenstein, M., Lyons, J. E. \& Runge, M. C. (2020). Using value of information to prioritize research needs for migratory bird management under climate change: a case study using federal land acquisition in the United States. Biological Reviews, 95, 11091130.

Saalfeld, S.T., Conway, W.C., Haukos, D.A. \& Johnson, W.P. (2011). Nest success of Snowy Plovers (Charadrius nivosus) in the southern high plains of Texas. Waterbirds, 34, 389399.

Saalfeld, S.T., Conway, W.C., Haukos, D.A. \& Johnson, W.P. (2012). Alleviation of nest thermal extremes by incubating Snowy Plovers in the Southern High Plains of Texas. Wader Study Group Bulletin, 119, 77-83.

Saalfeld, S. T., Conway, W. C., Haukos, D. A. \& Johnson, W. P. (2013a). Recent declines in apparent survival and survey counts of Snowy Plovers breeding in the Southern High Plains of Texas. The Wilson Journal of Ornithology, 125, 79-87. 
605 Saalfeld, S.T., Conway, W.C., Haukos, D.A. and Johnson, W.P. (2013b). Seasonal variation in 606 offspring sex ratio in the Snowy Plover. Western North American Naturalist, 60-71.

607 Sæther, B.-E., Sutherland, W. J. \& Engen, S. (2004). Climate influences on avian population 608 dynamics. Advances in Ecological Research, 35, 185-209.

609 Saunders, S. P., Farr, M. T., Wright, A. D., Bahlai, C. A., Ribeiro Jr, J. W., Rossman, S., 610 Sussman, A. L., Arnold, T. W. \& Zipkin, E. F. (2019). Disentangling data discrepancies 611 with integrated population models. Ecology, 100, e02714.

612 Schaub, M. \& Abadi, F. (2011). Integrated population models: a novel analysis framework for 613 deeper insights into population dynamics. Journal of Ornithology, 152, 227-237.

614 Schaub, M. \& Fletcher, D. (2015). Estimating immigration using a Bayesian integrated 615 population model: choice of parametrization and priors. Environmental and Ecological $616 \quad$ Statistics, 22, 535-549.

617 Schaub, M., Gimenez, O., Sierro, A. and Arlettaz, R. (2007). Use of integrated modeling to 618 enhance estimates of population dynamics obtained from limited data. Conservation 619 Biology, 21, 945-955.

620 Schaub, M., von Hirschheydt, J. and Grüebler, M.U., 2015. Differential contribution of demographic rate synchrony to population synchrony in barn swallows. Journal of Animal Ecology, 84, 1530-1541.

623 Schaub, M., Jakober, H. \& Stauber, W. (2013). Strong contribution of immigration to local population regulation: evidence from a migratory passerine. Ecology, 94, 1828-1838.

625 Schmidt, J.H. and Rattenbury, K.L., 2018. An open-population distance sampling framework for 626 assessing population dynamics in group-dwelling species. Methods in Ecology and 627 Evolution, 9, 936-945. 
628 Simmonds, M. P. \& Isaac, S. J. (2007). The impacts of climate change on marine mammals: 629 early signs of significant problems. Oryx, 41, 19-26.

630 Sofaer, H. R., Skagen, S. K., Barsugli, J. J., Rashford, B. S., Reese, G. C., Hoeting, J. A., Wood,

631

632

633

634

635

636

637

638

639

640

641

642

643

644

645

646

647

648

649

A. W. \& Noon, B. R. (2016). Projected wetland densities under climate change: habitat loss but little geographic shift in conservation strategy. Ecological Applications, 26, $1677-1692$.

Sorenson, L. G., Goldberg, R., Root, T. L. \& Anderson, M. G. (1998). Potential effects of global warming on waterfowl populations breeding in the northern Great Plains. Climatic Change, 40, 343-369.

Tavecchia, G., Besbeas, P., Coulson, T., Morgan, B. J. \& Clutton-Brock, T. H. (2009).

Estimating population size and hidden demographic parameters with state-space modeling. The American Naturalist, 173, 722-733.

Taylor, R. L., Walker, B. L., Naugle, D. E. \& Mills, L. S. (2012). Managing multiple vital rates to maximize greater sage-grouse population growth. The Journal of Wildlife Management, 76, 336-347.

Thomas, C. D., Cameron, A., Green, R. E., Bakkenes, M., Beaumont, L. J., Collingham, Y. C., Erasmus, B. F., De Siqueira, M. F., Grainger, A. \& Hannah, L. (2004). Extinction risk from climate change. Nature, 427, 145-148.

Todhunter, P. (1995). Hydroclimatic perspectives on waterfowl production in the North Dakota Prairie Pothole Region. Great Plains Research, 137-162.

Turchin, P. (2003). Complex population dynamics: a theoretical/empirical synthesis. Princeton University Press. 
650 Van de Pol, M., Vindenes, Y., Sæther, B.-E., Engen, S., Ens, B. J., Oosterbeek, K. \& Tinbergen, 651 J. M. (2010). Effects of climate change and variability on population dynamics in a 652 long-lived shorebird. Ecology, 91, 1192-1204.

653 Warriner, J.S., Warriner, J.C., Page, G.W. and Stenzel, L.E. (1986). Mating system and 654 reproductive success of a small population of polygamous Snowy Plovers. The Wilson $655 \quad$ Bulletin, 15-37.

656 Weegman, M. D., Arnold, T. W., Dawson, R. D., Winkler, D. W. \& Clark, R. G. (2017). 657 Integrated population models reveal local weather conditions are the key drivers of 658 population dynamics in an aerial insectivore. Oecologia, 185, 119-130.

659 Weegman, M.D., Arnold, T.W., Clark, R.G. \& Schaub, M. (2020). Partial and complete 660 dependency among data sets has minimal consequence on estimates from integrated 661 population models. Ecological Applications, e2258.

662 Williams, B. K., Nichols, J. D. \& Conroy, M. J. (2002). Analysis and management of animal 663 populations. Academic Press.

664 Williams, C. K., Ives, A. R. \& Applegate, R. D. (2003). Population dynamics across 665 geographical ranges: time-series analyses of three small game species. Ecology, 84, $666 \quad 2654-2667$.

667 Zhao, Q., Arnold, T. W., Devries, J. H., Howerter, D. W., Clark, R. G. \& Weegman, M. D. 668 (2019). Land use change increases climatic vulnerability of migratory birds: insights from 669 integrated population modelling. Journal of Animal Ecology, 88, 1625-1637.

670 Zhao, Q., Arnold, T. W., Devries, J. H., Howerter, D. W., Clark, R. G. \& Weegman, M. D. 671 (2020) Using integrated population models to prioritize region-specific conservation 
672 strategies under global change. Biological Conservation

$673 \quad$ https://doi.org/10.1016/j.biocon.2020.108832

674 Zhao, Q., Boomer, G. S. \& Royle, J. A. (2019). Integrated modeling predicts shifts in waterbird 675 population dynamics under climate change. Ecography, 42, 1470-1481.

676 Zhao, Q., Silverman, E., Fleming, K. \& Boomer, G. S. (2016). Forecasting waterfowl population 677 dynamics under climate change_-Does the spatial variation of density dependence and 678 environmental effects matter? Biological Conservation, 194, 80-88.

679 Zipkin, E. F. \& Saunders, S. P. (2018). Synthesizing multiple data types for biological 680 conservation using integrated population models. Biological Conservation, 217, 240-250. 681 
Figure 1

Study area

Figure 1. The position of the Great Plains in the contiguous US (inner panel), and the positions of our study sites in the Southern Great Plains in Texas (Muleshoe National Wildlife Refuge [NWR] and lakes A, B and C), New Mexico (Bitter Lake NWR), and Oklahoma (Salt Plains NWR).

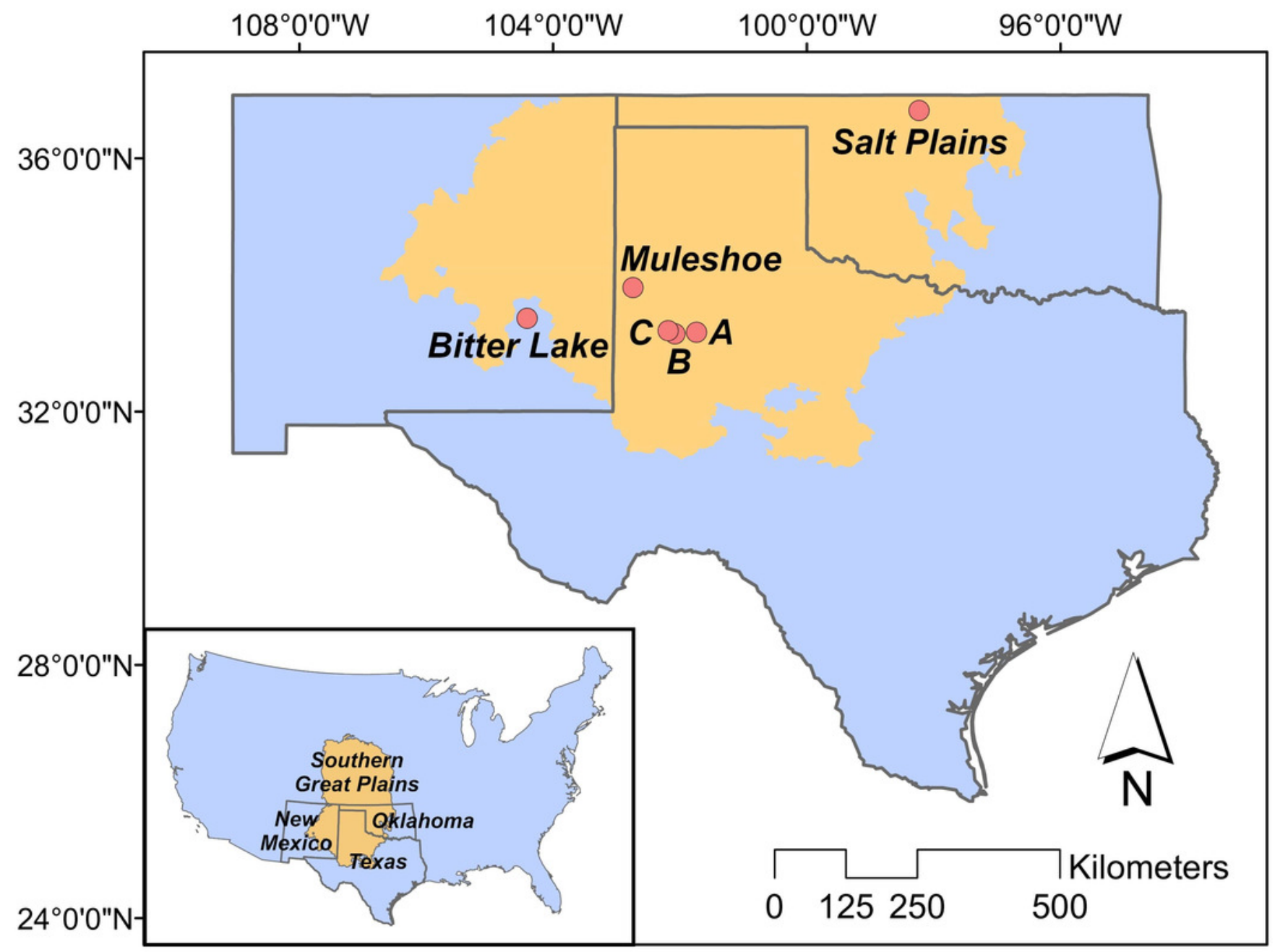


Figure 2

Data availability

Figure 2. The years in which each type of data (population survey, distance sampling, nest survey, and capture-recapture-resight) are available for each population (Texas, New Mexico, Oklahoma). 


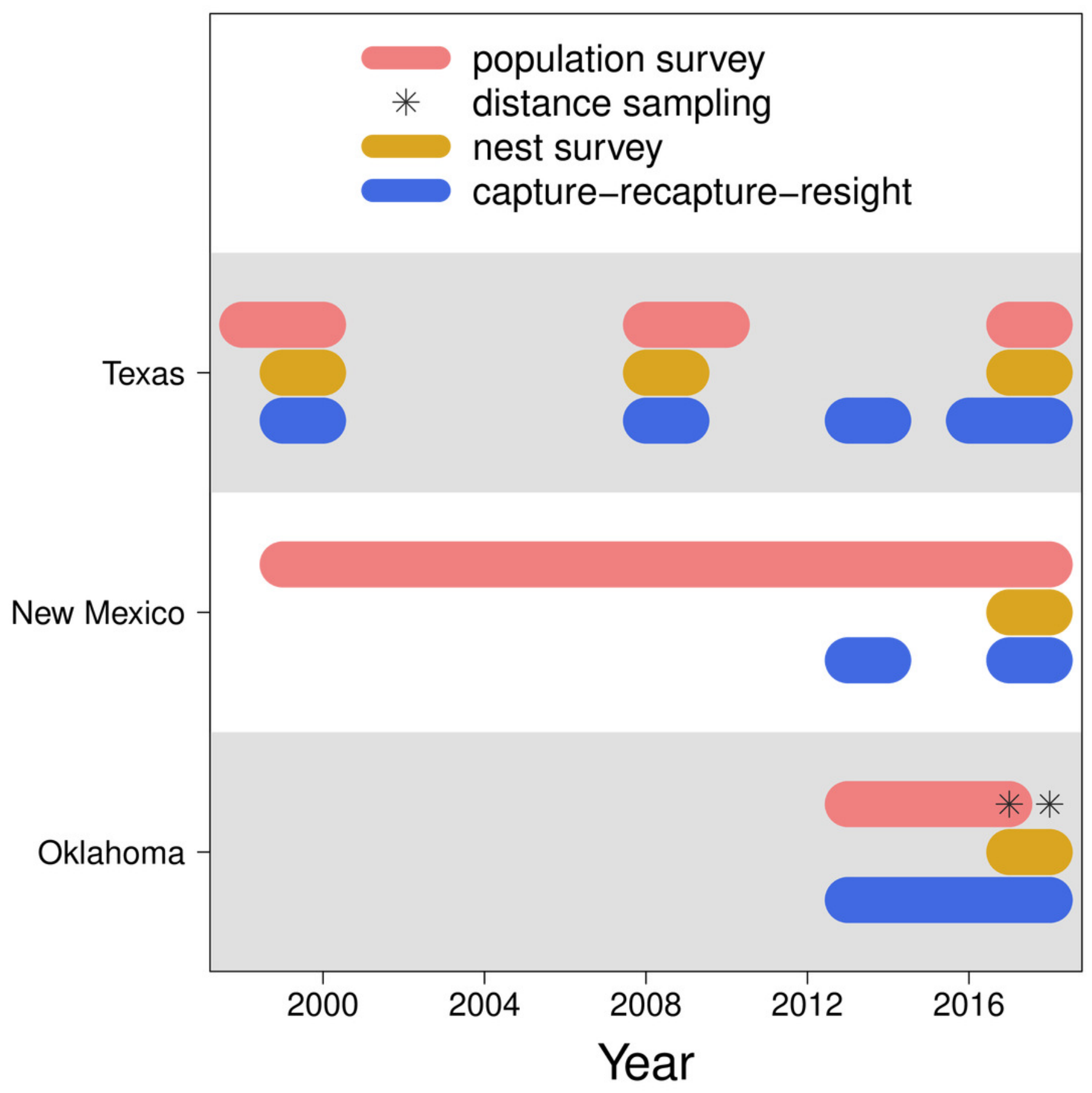


Figure 3

Population estimates

Figure 3. IPM estimated population trend (yellow line) and corresponding uncertainty gradient (purple strip) as well as population count data (red points) in Texas, New Mexico, and Oklahoma and distance sampling data (blue points) in Oklahoma.
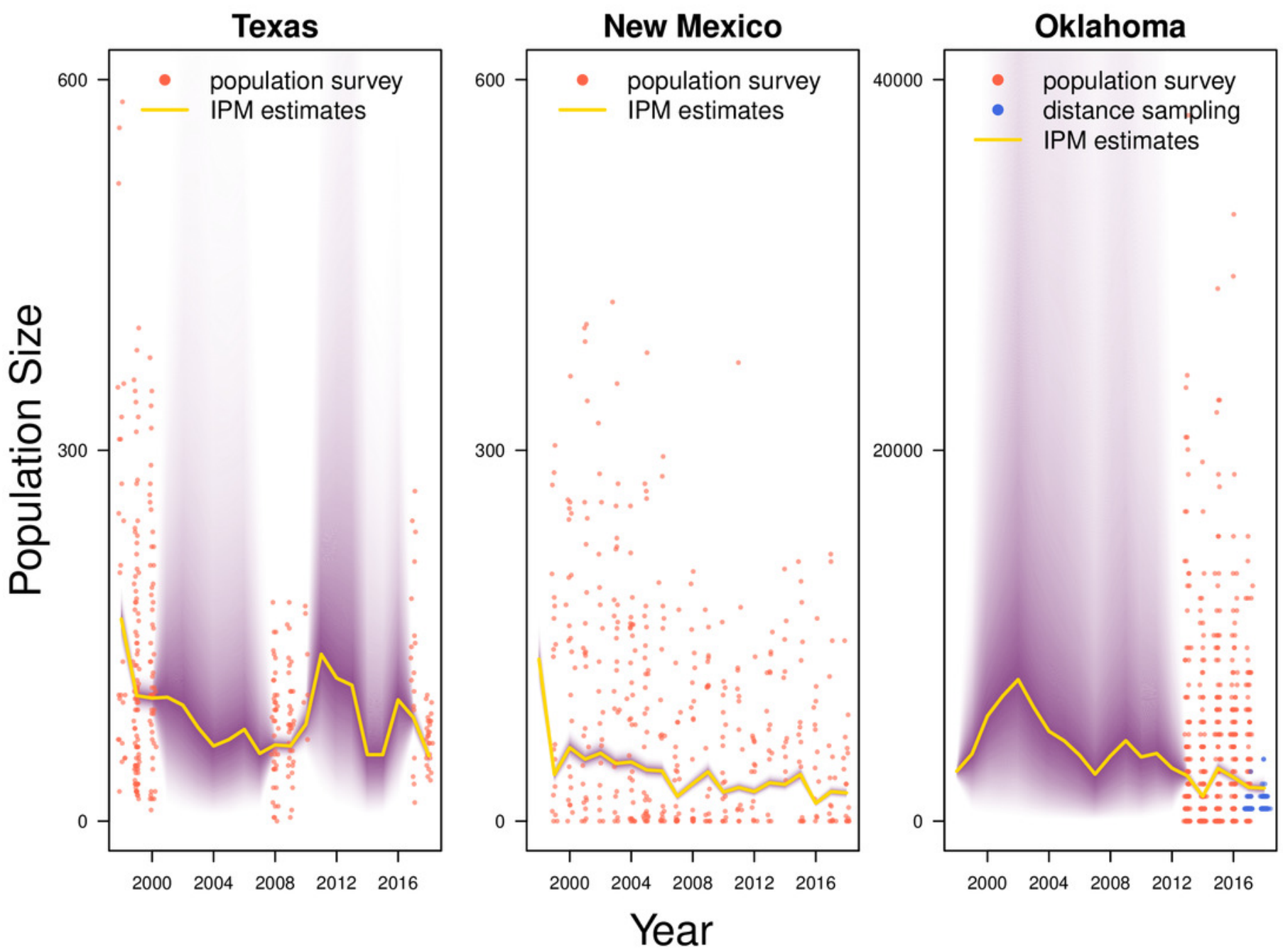
Figure 4

Productivity estimates

Figure 4. IPM estimated average clutch size and clutch fate (yellow line) and corresponding uncertainty gradient (purple strip) in Texas, New Mexico, and Oklahoma. Sample size (i.e., the number of nests surveyed; blue line) is also shown.

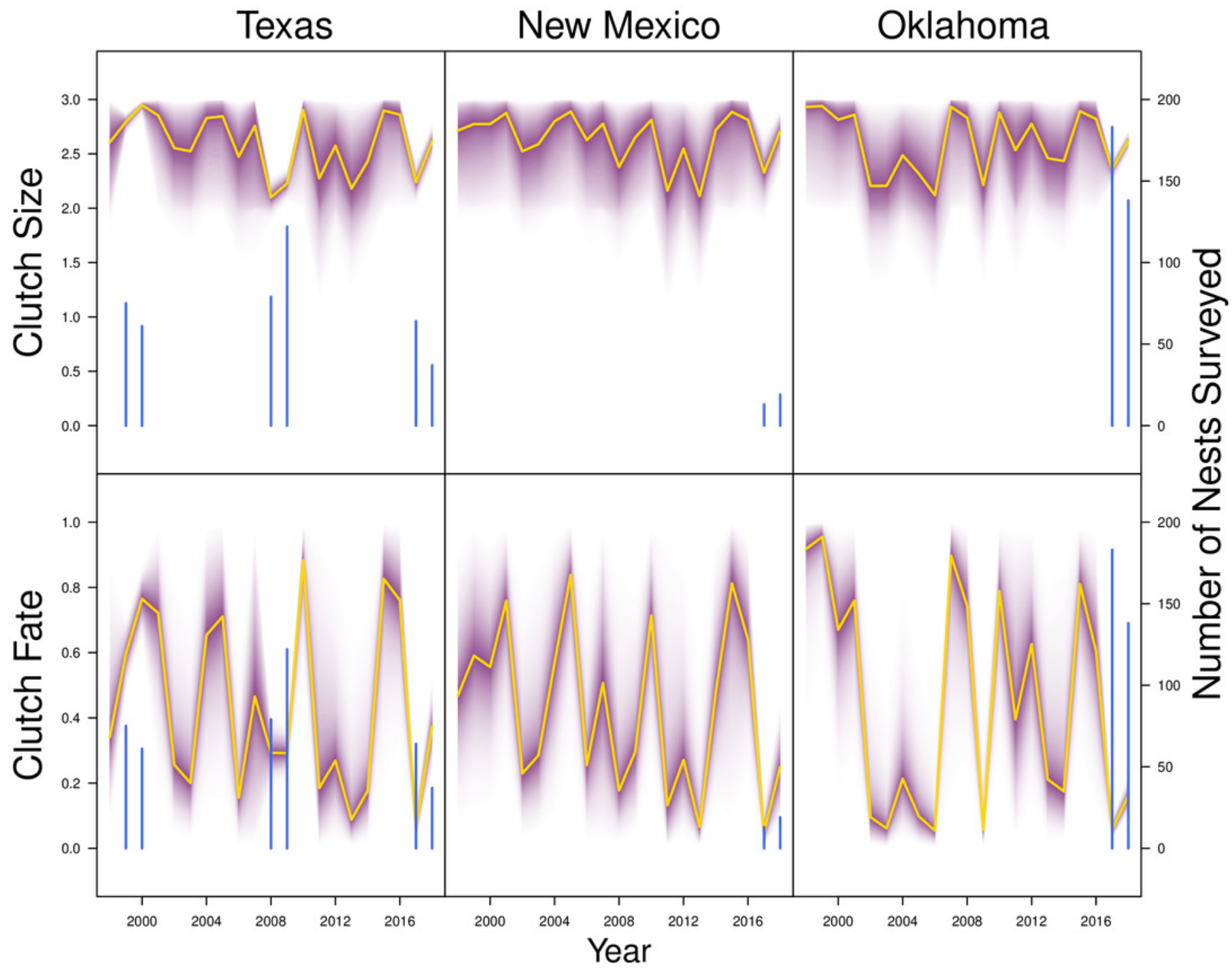




\section{Figure 5}

\section{Survival estimates}

Figure 5. IPM estimated apparent survival of adult female, adult male, juvenile female and juvenile male (yellow line) and corresponding uncertainty gradient (purple strip) in Texas, New Mexico, and Oklahoma. Sample size (i.e., the number of banded birds; blue line) is also shown.

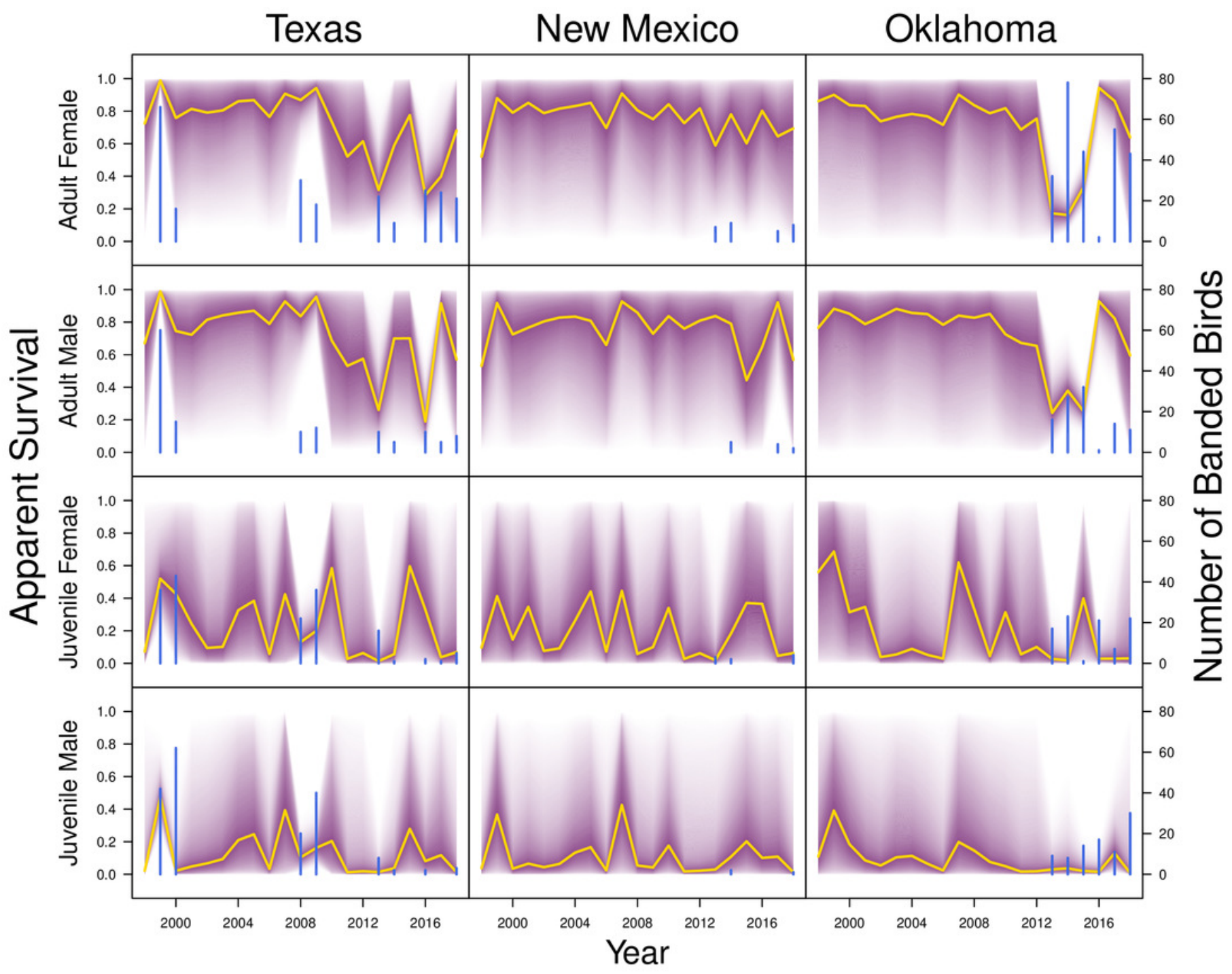




\section{Figure 6}

Demographic contributions

Figure 6. The relative independent contribution of average clutch size, clutch fate, and apparent survival of adult female, adult male, juvenile female, and juvenile male on snowy plover population growth in Texas, New Mexico, and Oklahoma. The violin plot shows the entire posterior distribution, while the embedded boxplot shows the median (white dot), 50\% Credible Interval (thick line), and 95\% Credible Interval (thin line). 


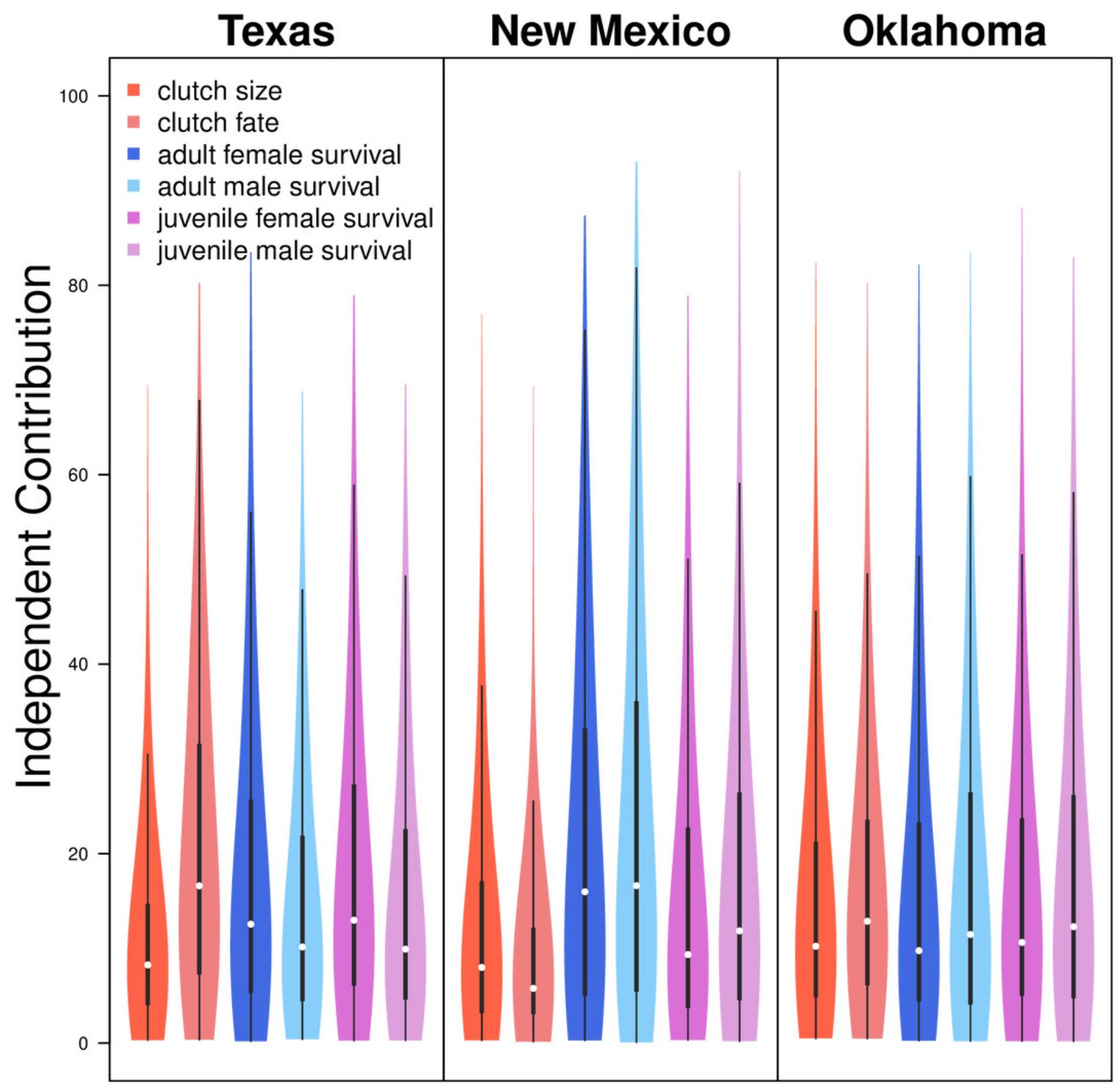




\section{Figure 7}

Drivers of productivity

Figure 7. Violin plots showing the posterior distributions of the slope parameters that represent the effect of Palmer drought severity index (PDSI), minimum temperature (min temp), and wind speed (wind) on productivity measures of snowy plover including the probability of a clutch size of 2 (C2) or 3 (C3), and clutch fate (FT). The violin plot shows the entire posterior distribution, while the embedded boxplot shows the median (white dot), 50\% Credible Interval (thick line), and 95\% Credible Interval (thin line). 


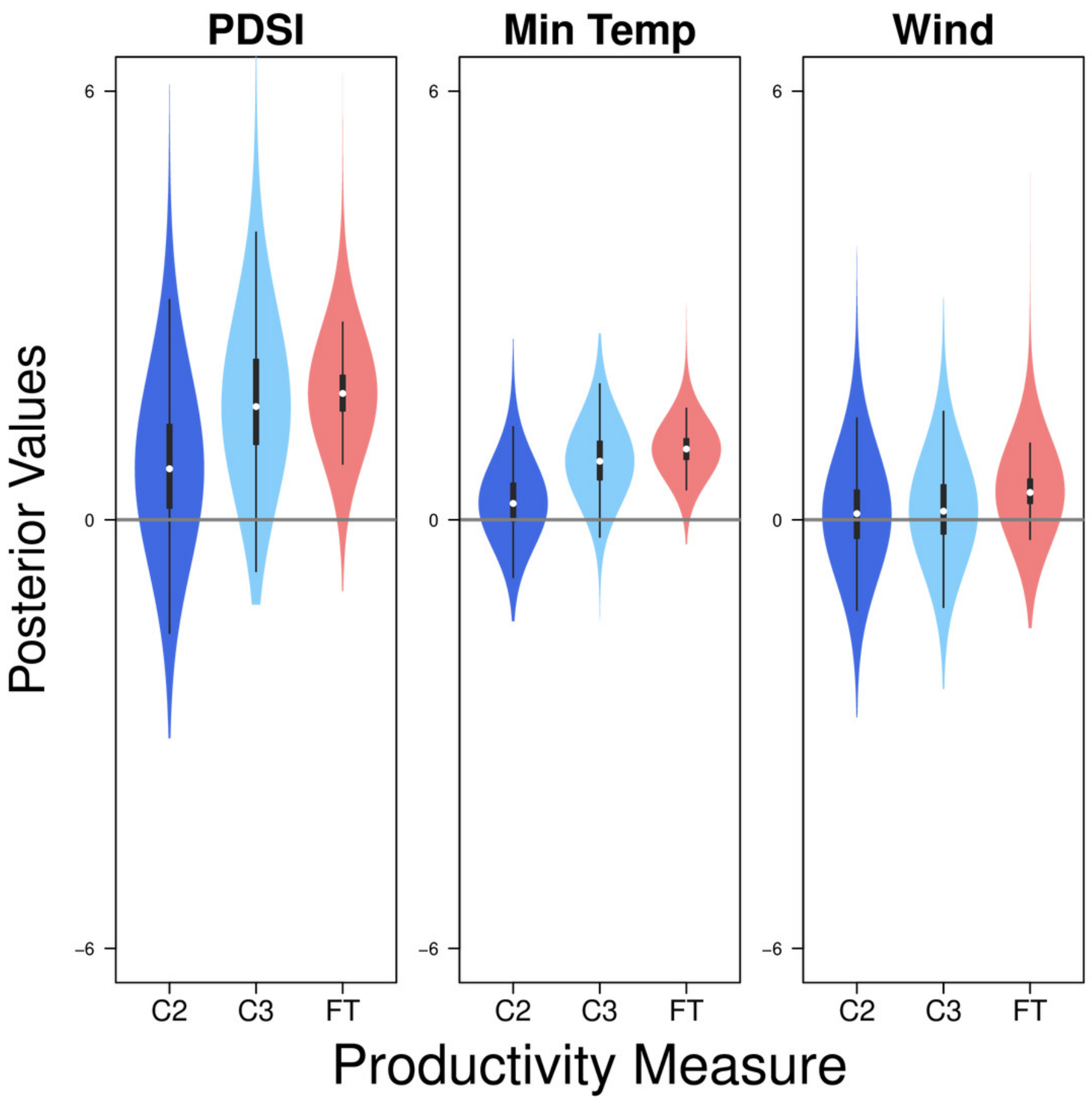


Figure 8

Response curves for productivity

Figure 8. Response curves showing the effects of Palmer drought severity index (PDSI) and minimum temperature (min temp) on average clutch size and clutch fate. The predicted relationships (yellow line) and corresponding uncertainty gradient (purple strip) for the estimated demographic parameters (blue point/line) are shown. 

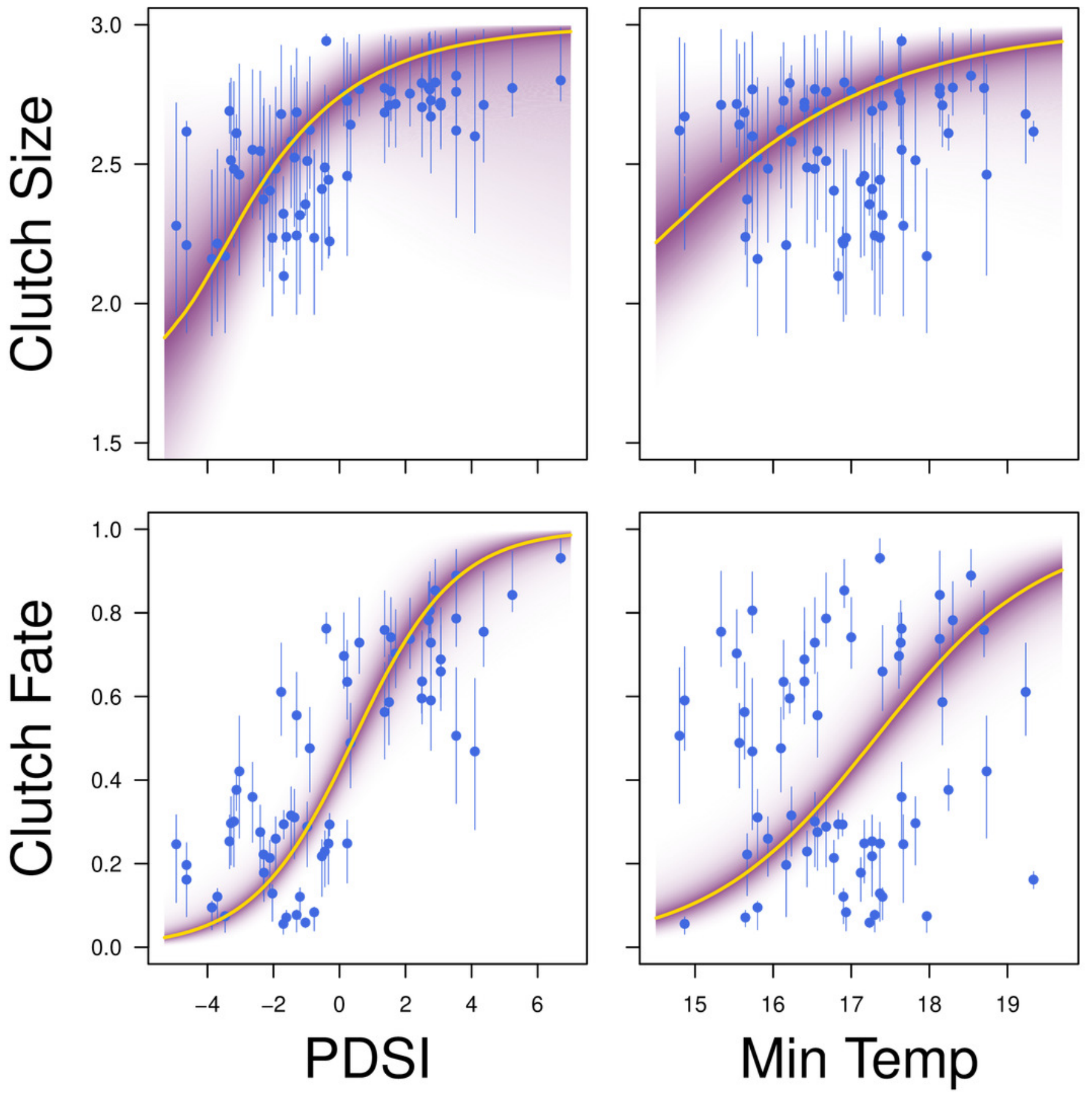


\section{Figure 9}

Drivers of survival

Figure 9. Violin plots showing the posterior distributions of the slope parameters that represent the effect of Palmer drought severity index (PDSI), minimum temperature (min temp), and wind speed (wind) on the apparent survival of adult female (AF), adult male (AM), juvenile female (JF), and juvenile male (JM) snowy plover. The violin plot shows the entire posterior distribution, while the embedded boxplot shows the median (white dot), 50\% Credible Interval (thick line), and 95\% Credible Interval (thin line). 


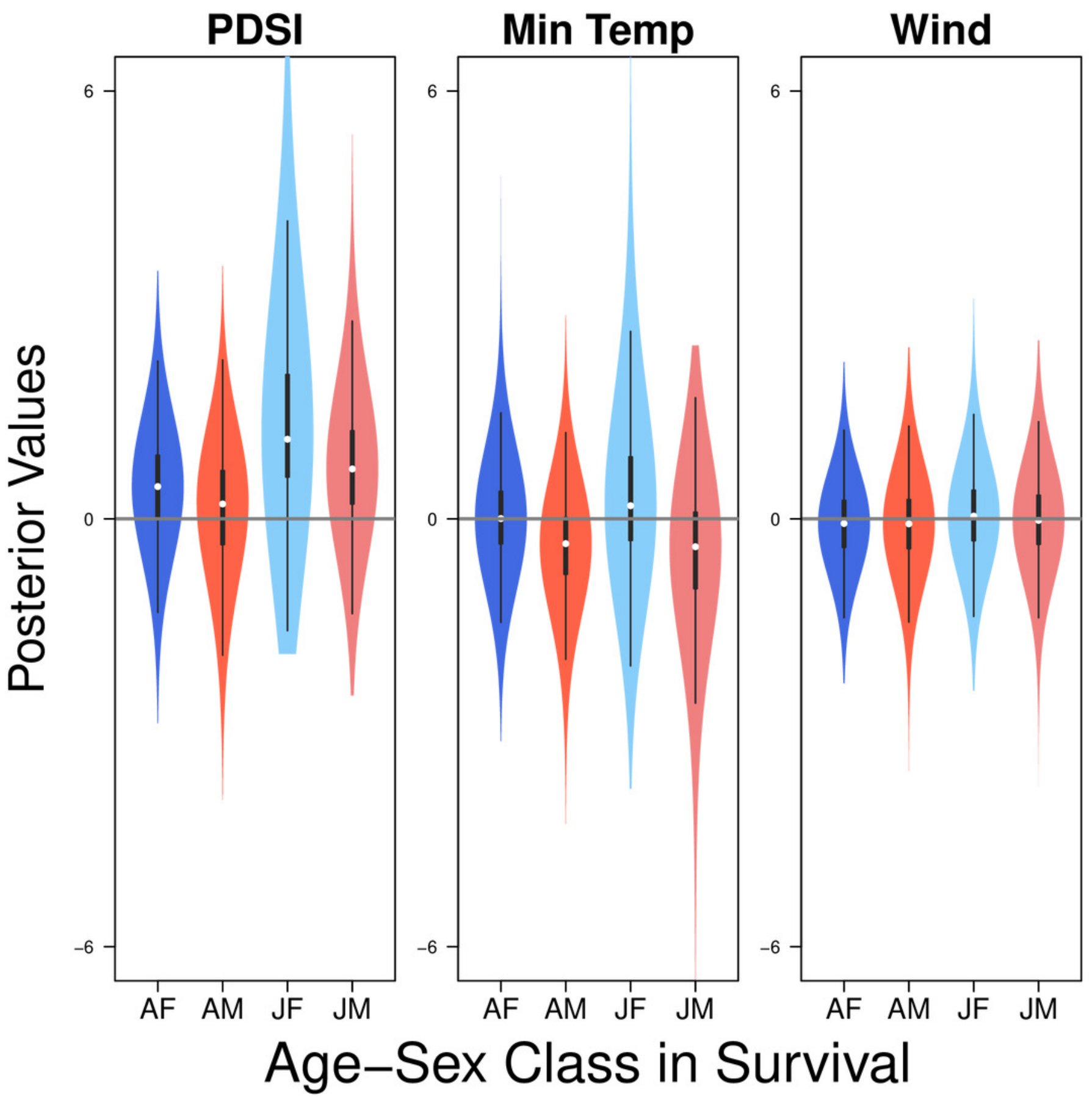

